\title{
Observation of events with a large rapidity gap in deep inelastic scattering at HERA
}

\section{ZEUS Collaboration}

M. Derrick, D. Krakauer, S. Magill, B. Musgrave, J. Repond, S. Repond, R. Stanek, R.L. Talaga, J. Thron

Argonne National Laboratory, Argonne, IL, USA ${ }^{47}$

F. Arzarello, R. Ayad ${ }^{1}$, G. Bari, M. Basile, L. Bellagamba, D. Boscherini, A. Bruni, G. Bruni, P. Bruni, G. Cara Romeo, G. Castellini ${ }^{2}$, M. Chiarini, L. Cifarelli, F. Cindolo, F. Ciralli,

A. Contin, S. D'Auria, C. Del Papa, F. Frasconi, P. Giusti, G. Iacobucci, G. Laurenti, G. Levi, Q. Lin, B. Lisowski, G. Maccarrone, A. Margotti, T. Massam, R. Nania, C. Nemoz, F. Palmonari, G. Sartorelli, R. Timellini, Y. Zamora Garcia ' , A. Zichichi

University and INFN Bologna, Bologna, Italy ${ }^{39}$

A. Bargende, J. Crittenden, K. Desch, B. Diekmann, T. Doeker, A. Frey, M. Geerts, G. Geitz, H. Hartmann, D. Haun, K. Heinloth, E. Hilger, H.-P. Jakob, S. Kramarczyk, M. Kückes ${ }^{3}$,

A. Mass, S. Mengel, J. Mollen, D. Monaldi ${ }^{4}$, H. Müsch ${ }^{5}$, E. Paul, R. Schattevoy, J.-L. Schneider, D. Schramm, R. Wedemeyer

Physikalisches Institut der Universität Bonn, Bonn, Federal Republic of Germany ${ }^{36}$

A. Cassidy, D.G. Cussans, N. Dyce, B. Foster, R. Gilmore, G.P. Heath, H.F. Heath, M. Lancaster, T.J. Llewellyn, J. Malos, C.J.S. Morgado, R.J. Tapper, S.S. Wilson, R. Yoshida H.H. Wills Physics Laboratory, University of Bristol, Bristol, UK ${ }^{46}$

R.R. Rau

Brookhaven National Laboratory, Upton, L.I., NY, USA ${ }^{47}$

M. Arneodo, M. Schioppa, G. Susinno

Calabria University, Physics Department and INFN, Cosenza, Italy ${ }^{39}$

A. Bernstein, A. Caldwell, I. Gialas, J.A. Parsons, S. Ritz, F. Sciulli ${ }^{6}$, P.B. Straub, L. Wai, S. Yang Columbia University, Nevis Labs., Irvington on Hudson, NY, USA ${ }^{48}$

P. Borzemski, J. Chwastowski ${ }^{7}$, A. Dwuraźny, A. Eskreys, Z. Jakubowski ${ }^{8}$, B. Nizioł, K. Piotrzkowski, M. Zachara, L. Zawiejski

Institute of Nuclear Physics, Cracow, Poland ${ }^{43}$ 
L. Adamczyk, B. Bednarek, K. Eskreys, K. Jeleń, D. Kisielewska, T. Kowalski,

E. Rulikowska-Zarȩbska, L. Suszycki, J. Zając

Faculty of Physics and Nuclear Techniques, Academy of Mining and Metallurgy, Cracow, Poland ${ }^{43}$

T. Kędzierski, A. Kotański, M. Przybycień

Jagellonian University, Department of Physics, Cracow, Poland 43

L.A.T. Bauerdick, U. Behrens, J.K. Bienlein, S. Böttcher, C. Coldewey, A. Dannemann, G. Drews, P. Erhard ${ }^{9}$, M. Flasiński ${ }^{10}$, I. Fleck, R. Gläser ${ }^{11}$, P. Göttlicher, B. Gutjahr, T. Haas, L. Hagge, W. Hain, D. Hasell, H. Hultschig, G. Jahnen ${ }^{12}$, P. Joos, M. Kasemann, R. Klanner, W. Koch, L. Köpke, U. Kötz, H. Kowalski, W. Kröger, J. Krüger, J. Labs, A. Ladage, B. Löhr, M. Löwe, D. Lüke, J. Mainusch, O. Manczak, M. Momayezi ${ }^{13}$, J.S.T. Ng, S. Nickel, D. Notz, K.-U. Pösnecker ${ }^{14}$, M. Rohde, J. Roldán ${ }^{15}$, E. Ros ${ }^{7}$, U. Schneekloth, J. Schroeder, W. Schulz, F. Selonke, E. Stiliaris ${ }^{15}$, E. Tscheslog ${ }^{17}$, T. Tsurugai, W. Vogel ${ }^{17}$, G. Wolf, C. Youngman Deutsches Elektronen-Synchrotron DESY, Hamburg, Federal Republic of Germany

H.J. Grabosch, A. Leich, A. Meyer, C. Rethfeldt, S. Schlenstedt DESY-Zeuthen, Institut für Hochenergiephysik, Zeuthen, Federal Republic of Germany

G. Barbagli, A. Francescato, M. Nuti, P. Pelfer

University and INFN, Florence, Italy ${ }^{39}$

G. Anzivino, R. Casaccia, S. De Pasquale, S. Qian, L. Votano INFN, Laboratori Nazionali di Frascati, Frascati, Italy ${ }^{39}$

A. Bamberger, A. Freidhof, T. Poser, S. Söldner-Rembold, G. Theisen, T. Trefzger Physikalisches Institut der Universität Freiburg, Freiburg, Federal Republic of Germany ${ }^{36}$

N.H. Brook, P.J. Bussey, A.T. Doyle, J.R. Forbes, V.A. Jamieson, C. Raine, D.H. Saxon Department of Physics and Astronomy, University of Glasgow, Glasgow, UK ${ }^{\mathbf{4}}$

H. Brückmann ${ }^{18}$, G. Gloth, U. Holm, H. Kammerlocher, B. Krebs, T. Neumann, K. Wick Hamburg University, Ist Institute of Experimental Physics, Hamburg, Federal Republic of Germany ${ }^{36}$

A. Fürtjes, E. Lohrmann, J. Milewski ${ }^{19}$, M. Nakahata ${ }^{20}$, N. Pavel, G. Poelz, W. Schott, J. Terron ${ }^{15}, \mathrm{~F}$. Zetsche

Hamburg University, IInd Institute of Experimental Physics, Hamburg, Federal Republic of Germany ${ }^{36}$

T.C. Bacon, R. Beuselinck, I. Butterworth, E. Gallo, V.L. Harris, D.B. Miller, A. Prinias, J.K. Sedgbeer, A. Vorvolakos, A. Whitfield Imperial College London, High Energy Nuclear Physics Group, London, UK ${ }^{46}$ 
T. Bienz, H. Kreutzmann, U. Mallik, E. McCliment, M. Roco, M.Z. Wang

University of Iowa, Physics and Astronomy Department, Iowa City, USA ${ }^{4}$ ?

P. Cloth, D. Filges

Forschungszentrum Jülich, Institut für Kernphysik, Jülich. Federal Republic of Germany

S.H. An, S.M. Hong, C.O. Kim, T.Y. Kim, S.W. Nam, S.K. Park, M.H. Suh, S.H. Yon

Korea University, Seoul, Korea ${ }^{41}$

R. Imlay, S. Kartik, H.-J. Kim, R.R. McNeil, W. Metcalf, V.K. Nadendla

Louisiana State University, Department of Physics and Astronomy, Baton Rouge, LA, USA 47

F. Barreiro ${ }^{21}$, G. Cases, L. Hervás ${ }^{22}$, L. Labarga ${ }^{22}$, J. del Peso, J.F. de Trocóniz ${ }^{23}$

Univer. Autónoma Madrid, Depto de Física Teórica, Madrid, Spain ${ }^{45}$

F. Ikraiam, J.K. Mayer, G.R. Smith

University of Manitoba, Department of Physics, Winnipeg, Manitoba, Canada 34

F. Corriveau, D.J. Gilkinson, D.S. Hanna ${ }^{6}$, J. Hartmann, L.W. Hung, J.N. Lim, R. Meijer Drees, J.W. Mitchell, P.M. Patel, L.E. Sinclair, D.G. Stairs, M. St.Laurent, R. Ullmann

McGill University, Department of Physics, Montreal, Quebec, Canada ${ }^{34,35}$

G.L. Bashindzhagyan, P.F. Ermolov, L.K. Gladilin, Y.A. Golubkov, V.A. Kuzmin,

E.N. Kuznetsov, A.A. Savin, A.G. Voronin, N.P. Zotov

Moscow State University, Institute of Nuclear Pysics, Moscow, Russia 44

S. Bentvelsen, M. Botje, A. Dake, J. Engelen, P. de Jong, M. de Kamps, P. Kooijman, A. Kruse, H. van der Lugt, V. O'Dell, A. Tenner, H. Tiecke, H. Uijterwaal ${ }^{24}$, M. Vreeswijk, L. Wiggers, E. de Wolf, R. van Woudenberg

NIKHEF-Amsterdam, Amsterdam, Netherlands ${ }^{42}$

B. Bylsma, L.S. Durkin, K. Honscheid, C. Li, T.Y. Ling, K.W. McLean, W.N. Murray,

I.H. Park, T.A. Romanowski ${ }^{25}$, R. Seidlein

Ohio State University, Physics Department, Columbus, OH, USA ${ }^{47}$

G.A. Blair, A. Byrne, R.J. Cashmore, A.M. Cooper-Sarkar, R.C.E. Devenish, D.M. Gingrich ${ }^{26}$, P.M. Hallam-Baker ${ }^{7}$, N. Harnew, T. Khatri, K. R. Long, P. Luffman, I. McArthur, P. Morawitz, J. Nash, S.J.P. Smith ${ }^{27}$, N.C. Roocroft, F.F. Wilson

Department of Physics, University of Oxford, Oxford, $U K^{46}$

G. Abbiendi, R. Brugnera, R. Carlin, F. Dal Corso, M. De Giorgi, U. Dosselli, F. Gasparini, S. Limentani, M. Morandin, M. Posocco, L. Stanco, R. Stroili, C. Voci

Dipartimento di Fisica dell' Università and INFN, Padova, Italy ${ }^{39}$ 
J.M. Butterworth, J. Bulmahn, G. Feild, B.Y. Oh ${ }^{28}$, J. Whitmore ${ }^{29}$

Pennsylvania State University, Department of Physics, University Park, PA, USA ${ }^{48}$

U. Contino, G. D’Agostini, M. Guida ${ }^{30}$, M. Iori, S.M. Mari, G. Marini, M. Mattioli, A. Nigro Dipartimento di Fisica, Università 'La Sapienza' and INFN, Rome, Italy ${ }^{39}$

J.C. Hart, N.A. McCubbin, K. Prytz, T.P. Shah, T.L. Short

Rutherford Appleton Laboratory, Chilton, Didcot, Oxon, $U K^{46}$

E. Barberis, N. Cartiglia, C. Heusch, B. Hubbard, J. Leslie, W. Lockman, K. O’Shaughnessy, H.F. Sadrozinski, A. Seiden, D. Zer-Zion

University of California, Santa Cruz, CA, USA ${ }^{47}$

E. Badura, J. Biltzinger, R.J. Seifert, A.H. Walenta, G. Zech

Fachbereich Physik der Universität-Gesamthochschule Siegen, Siegen, Federal Republic of Germany ${ }^{36}$

S. Dagan ${ }^{31}$, A. Levy

School of Physics, Tel-Aviv University, Tel Aviv, Israel ${ }^{38}$

T. Hasegawa, M. Hazumi, T. Ishii, S. Kasai ${ }^{32}$, M. Kuze, S. Mine, Y. Nagasawa, T. Nagira, M. Nakao, H. Okuno, I. Suzuki, K. Tokushuku, S. Yamada, Y. Yamazaki

Institute for Nuclear Study, University of Tokyo, Tokyo, Japan ${ }^{40}$

M. Chiba, R. Hamatsu, T. Hirose, S. Kitamura, S. Nagayama, Y. Nakamitsu

Tokyo Metropolitan University, Department of Physics, Tokyo, Japan 40

R. Cirio, M. Costa, M.I. Ferrero, L. Lamberti, S. Maselli, C. Peroni, A. Solano, A. Staiano

Università di Torino, Dipartimento di Fisica Sperimentale and INFN, Torino, Italy 39

M. Dardo

Facultá di Scienze, University of Torino, Alessandria, and INFN-Sezione di Torino, Torino, Italy 39

D.C. Bailey, D. Bandyopadhyay, F. Benard, S. Bhadra, M. Brkic, B.D. Burow, F.S. Chlebana, M.B. Crombie, G.F. Hartner, G.M. Levman, J.F. Martin, R.S. Orr, J.D. Prentice,

C.R. Sampson, G.G. Stairs, R.J. Teuscher, T.-S. Yoon

University of Toronto, Department of Physics, Toronto, Ont., Canada ${ }^{34}$

F.W. Bullock, C.D. Catterall, J.C. Giddings, T.W. Jones, A.M. Khan, J.B. Lane, P.L. Makkar, D. Shaw, J. Shulman

University College London, Physics and Astronomy Department, London, UK ${ }^{46}$ 
K. Blankenship, J. Kochocki, B. Lu, L.W. Mo

Virginia Polytechnic Institute and State University, Physics Department, Blacksburg, VA, USA 48

K. Charchuła, J. Ciborowski, J. Gajewski, G. Grzelak, M. Kasprzak, M. Krzyżanowski,

K. Muchorowski, R.J. Nowak, J.M. Pawlak, A. Stopczyński, T. Tymieniecka, R. Walczak, A.K. Wróblewski, J.A. Zakrzewski, A.F. Żarnecki

Warsaw University, Institute of Experimental Physics, Warsaw, Poland ${ }^{43}$

\section{Adamus}

Institute for Nuclear Studies, Warsaw, Poland ${ }^{43}$

H. Abramowicz ${ }^{19}$, Y. Eisenberg, C. Glasman ${ }^{33}$, U. Karshon ${ }^{31}$, A. Montag ${ }^{31}$, D. Revel, A. Shapira Weizmann Institute, Nuclear Physics Department, Rehovot, Israel ${ }^{37}$

C. Foudas, C. Fordham, R.J. Loveless, A. Goussiou, I. Ali, B. Behrens, S. Dasu, D.D. Reeder, W.H. Smith, S. Silverstein

University of Wisconsin, Department of Physics, Madison, WI, USA 47

W.R. Frisken, K.M. Furutani, Y. Iga

York University, Department of Physics, North York, Ont., Canada ${ }^{34}$

Received 16 July 1993

Editor: K. Winter

In deep inelastic, neutral current scattering of electrons and protons at $\sqrt{s}=296 \mathrm{GeV}$, we observe in the ZEUS detector events with a large rapidity gap in the hadronic final state. They occur in the region of small Bjorken $x$ and are observed up to $Q^{2}$ of $100 \mathrm{GeV}^{2}$. They account for about $5 \%$ of the events with $Q^{2} \geqslant 10 \mathrm{GeV}^{2}$. Their general properties are inconsistent with the dominant mechanism of deep inelastic scattering, where color is transferred between the scattered quark and the proton remnant, and suggest that the underlying production mechanism is the diffractive dissociation of the virtual photon.

Supported by Worldlab, Lausanne, Switzerland. Also at IROE Florence, Italy.

Now at TRIUMF, Vancouver.

Now at University of Bologna.

Now a self-employed consultant.

Now at DESY as Alexander von Humboldt Fellow.

Now at CERN.

Now at DESY.

Now at IST GmbH, Darmstadt.

10 On leave from Jagellonian University, Cracow.

11 Now at Martin \& Associates, Hamburg.

12 Now at Harry Hoffmann, Fitzbek.

13 Now at University of Minnesota, Minneapolis.

14 Now at Lufthansa, Frankfurt.

15 Supported by the European Community.

16 Now at Integrata, Frankfurt.

17 Now at Blohm \& Voss, Hamburg.
18 Deceased.

19 On leave from Warsaw University.

20 Now at Institute for Cosmic Ray Research, University of Tokyo.

21 On leave of absence at DESY, supported by DGICYT.

22 Partially supported by Comunidad Autónoma de Madrid, Spain.

23 Supported by Fundación Banco Exterior.

24 Now at SSC, Dallas.

25 Now at Department of Energy, Washington.

26 Now at Centre for Subatomic Research, University of Alberta, Canada and TRIUMF, Vancouver, Canada.

27 Now with McKinsey Consultants, Sidney, Australia.

28 On leave and supported by DESY 1992-93.

29 On leave and supported by DESY 1991-92.

30 Permanent address: Dipartimento di Fisica, Università di Salerno, Italy. 


\section{Introduction}

The dominant mechanism of deep inelastic electron proton scattering (DIS) is the scattering of the incident lepton from a colored quark (see fig. 1a). The color transfer between the struck quark and the proton remnant is responsible for populating the rapidity interval between them with final state hadrons. This picture predicts that DIS events observed in HERA experiments should have substantial energy deposits at angles close to the proton beam direction. Indeed, the energy flow distributions from the bulk of DIS events are consistent with this paradigm [1,2].

In this paper we report the first observation of a class of DIS events in which the hadronic energy deposit closest to the proton beam direction is at a large

31 Supported by the MINERVA Gesellschaft für Forschung $\mathrm{GmbH}$.

32 Now at Hiroshima National College of Maritime Technology.

33 Supported by the DAAD - Deutscher akademischer Austauschdienst.

34 Supported by the Natural Sciences and Engineering Research Council of Canada.

35 Supported by the FCAR of Quebec, Canada.

36 Supported by the German Federal Ministry for Research and Technology (BMFT).

37 Supported by the MINERVA Gesellschaft für Forschung GmbH, by the Israel Ministry of Energy, and by the Israel Academy of Science.

38 Supported by the Israel Ministry of Energy, and by the German Israeli Foundation.

39 Supported by the Italian National Institute for Nuclear Physics (INFN).

40 Supported by the Japanese Ministry of Education, Science and Culture (the Monbusho) and its grants for Scientific Research.

41 Supported by the Korean Ministry of Education and Korea Science and Engineering Foundation.

42 Supported by the Netherlands Foundation for Research on Matter (FOM).

43 Supported by the Polish State Committee for Scientific Research (grant No. 204209101).

44 Supported by the German Federal Ministry for Research and Technology (BMFT), the Volkswagen Foundation, and the Deutsche Forschungsgemeinschaft.

45 Supported by the Spanish Ministry of Education and Science through funds provided by CICYT.

46 Supported by the UK Science and Engineering Research Council.

47 Supported by the US Department of Energy.

48 Supported by the US National Science Foundation. angle. Described in terms of pseudorapidity, $\eta=$ $-\ln \tan (\theta / 2)$, these events exhibit a sizeable difference between the pseudorapidity of the smallest detector angle ${ }^{\# 1}\left(\theta=1.5^{\circ}, \eta=4.3\right)$ and the pseudorapidity of the hadrons observed closest to the proton direction. These "large rapidity gap" events are not described by standard QCD inspired fragmentation models [3]. Their general characteristics are compatible with those expected from diffractive dissociation involving pomeron exchange.

Until recently, Regge theory and perturbative QCD have been subjects without much overlap, except for predictions regarding the $x \rightarrow 0$ behavior of structure functions [4]. The Regge phenomenology that successfully describes the energy dependence of the total cross sections and the properties of elastic and diffractive production in hadron-hadron interactions [5] involves the exchange of the pomeron. However, the present understanding of the nature of the pomeron is poor despite a wealth of data [6]. Ingelman and Schlein [7] suggested that the pomeron may have a partonic structure which could be probed in hard diffractive dissociation. Collider data from the UA8 Collaboration [8] give strong evidence for high transverse momentum jets in diffractively produced high mass systems which can be understood as the hard scattering from partons within the pomeron. The concept of the pomeron structure function has been studied in terms of perturbative QCD [4,9-15]. It has been suggested that the pomeron structure could be probed with a virtual photon at HERA $[7,16,17]$ and that the experimental signature of a pomeron exchange would consist of a quasi-elastically scattered proton, well separated in rapidity from the remaining hadronic system as illustrated in fig. $1 \mathrm{~b}$. The events reported here have such a rapidity gap.

The data were collected during fall 1992 with the ZEUS detector at HERA for collisions of $820 \mathrm{GeV}$ protons with $26.7 \mathrm{GeV}$ electrons. The data sample used in this analysis corresponds to an integrated luminosity of $24.7 \mathrm{nb}^{-1}$.

\#1 In the ZEUS coordinate system the polar angle $\theta$ is measured with respect to the $Z$ axis pointing along the direction of the incoming proton. 
a)

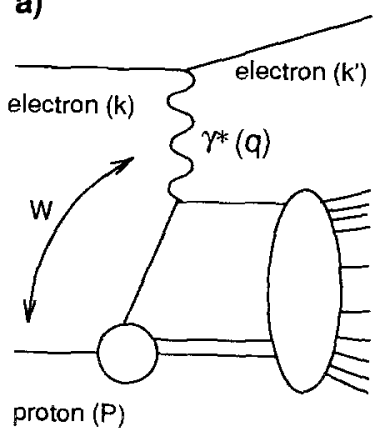

b)

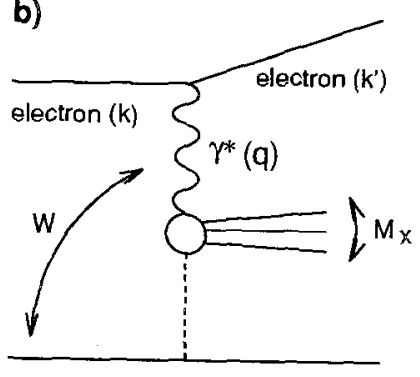

proton $(P)$

$N\left(P^{\prime}\right)$

c)

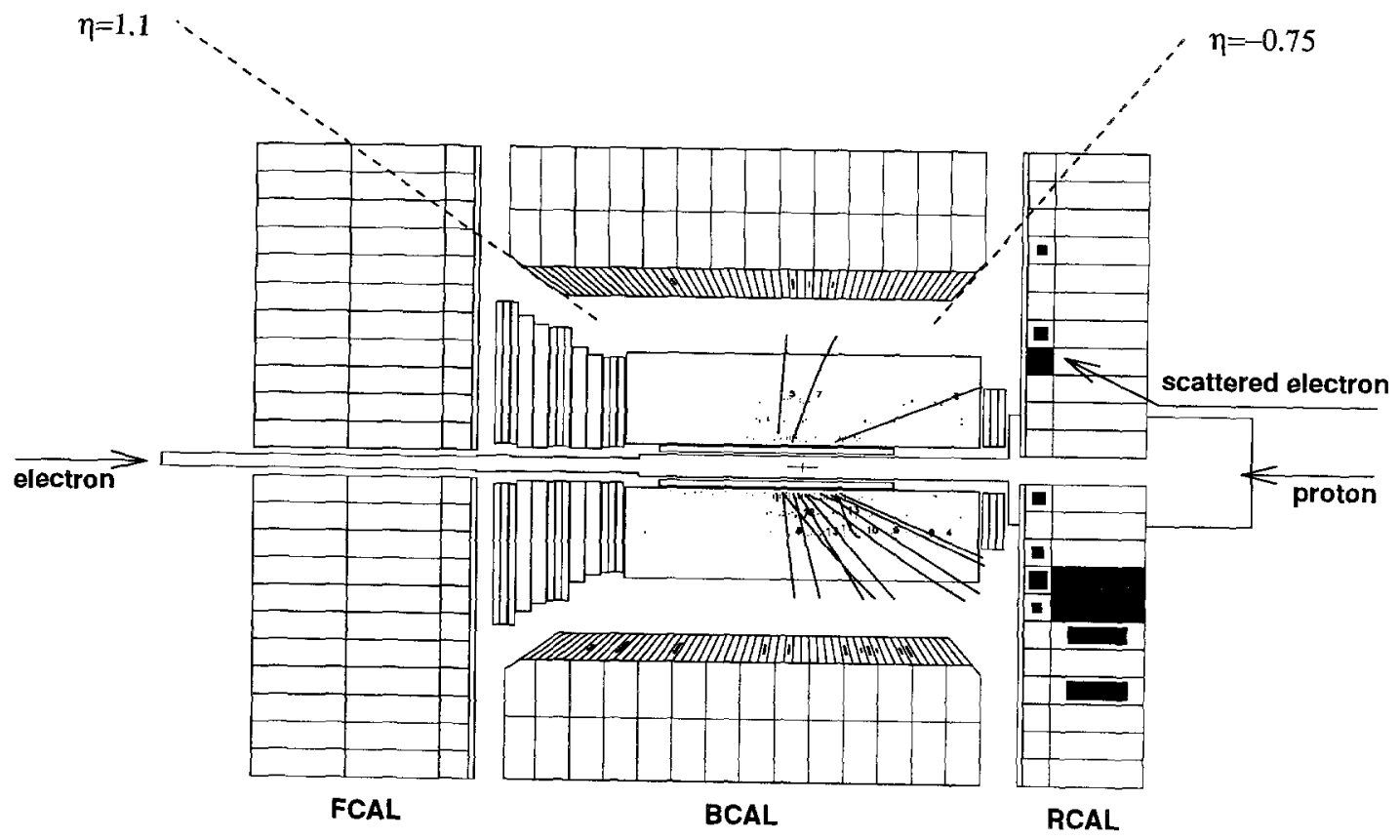

Fig. 1. (a) Schematic diagram describing particle production in deep inelastic electron proton scattering. (b) Schematic diagram describing particle production by diffractive dissociation in a deep inelastic ep interaction. $W$ is the center of mass energy of the $\gamma^{*} p$ system and $M_{\mathrm{X}}$ is the invariant mass of the hadronic system measured in the detector. $N$ represents a proton or low-mass nuclear system. (c) Schematic view of the ZEUS calorimeter and central tracking. Overlaid is an event with a large rapidity gap. Tracks are detected in the central tracking chamber and energy deposits are observed in the calorimeter. The electron is detected in the rear direction (RCAL).

\section{Experimental environment}

The ZEUS detector has been described elsewhere [18]. The hermetic uranium-scintillator calorimeter (CAL), the central tracking detector and the vertex detector were the main components used for this analysis.
The calorimeter consists of three parts (see fig. 1c): RCAL covering the backward pseudorapidity range $(-3.8<\eta<-0.75)$, BCAL covering the central region $(-0.75<\eta<1.1)$, and FCAL covering the forward region $(1.1<\eta<4.3)$. The scintillator tiles form towers that are longitudinally segmented into electromagnetic (EMC) and hadronic (HAC) cells. 
Characteristic transverse sizes are $5 \mathrm{~cm} \times 20 \mathrm{~cm}$ for the EMC cells of FCAL and BCAL and $10 \mathrm{~cm} \times 20$ $\mathrm{cm}$ for those in the RCAL. The HAC cells are typically $20 \mathrm{~cm} \times 20 \mathrm{~cm}$ in the transverse dimensions. Holes of $20 \mathrm{~cm} \times 20 \mathrm{~cm}$ in the center of FCAL and RCAL are required to accommodate the HERA beam pipe. The resulting solid angle coverage of the calorimeter is $99.7 \%$ of $4 \pi$.

The calorimeter readout provides precise energy and time measurements for the 5918 cells, each of which is instrumented with two photomultipliers. On average less than 0.1 percent of the cells were without operational readout. The calorimeter noise, which is dominated by the uranium radioactivity, is typically $15 \mathrm{MeV}$ in the EMC cells and $25 \mathrm{MeV}$ in the HAC cells. A cut of $60 \mathrm{MeV}$ for the EMC cells and $110 \mathrm{MeV}$ for the larger HAC cells reduces the influence of noise on the measurement of kinematic variables.

The tracking information was used for most events to determine the $Z$ coordinate of the event vertex with a resolution of $4 \mathrm{~cm}$. The time measured in the calorimeter can also be used to determine the $Z$ coordinate of the vertex with a resolution of $10 \mathrm{~cm}$. If neither was available the vertex was set to the nominal $Z$ coordinate of the interaction point, leading to an uncertainty of $25 \mathrm{~cm}$.

Data were collected while HERA collided nine electron and proton bunches. During the same period, single unpaired bunches of electrons and protons circulated, which permitted a measurement of beam associated backgrounds.

\section{Kinematics of deep inelastic events}

The kinematic variables used to describe deep inelastic scattering events are the following: $Q^{2}$, the negative of the squared four-momentum transfer carried by the virtual photon ${ }^{\# 2}, \gamma^{*}$,

$Q^{2}=-q^{2} \equiv-\left(k-k^{\prime}\right)^{2}$,

where $k$ and $k^{\prime}$ are the four-momentum vectors of the initial and final state electron, respectively; $y$, the

\#2 In the momentum transfer range accessible with our integrated luminosity, the electron-proton interactions are described by the exchange of a virtual photon. variable which describes the energy transfer to the hadronic final state,

$y \equiv(q \cdot P) /(k \cdot P)$

where $P$ is the four-momentum vector of the incoming proton; $x$, the Bjorken variable,

$x \equiv Q^{2} /(2 q \cdot P)=Q^{2} / y s$,

where $s$ is the center-of-mass energy squared of the $e p$ system; and $W$, the center-of-mass energy of the $\gamma^{*} p$ system,

$W^{2} \equiv(q+P)^{2}=\frac{Q^{2}(1-x)}{x}+M_{p}^{2}$,

where $M_{p}$ is the mass of the proton.

The kinematic variables can be determined from either the leptonic or the hadronic system. Studies of the HERA kinematics have shown [19] that it is sometimes advantageous to use a mixed set of variables. We chose to use the so called "double angle" method. The relevant quantities will be denoted by the subscript DA. Here all event variables are derived from the scattering angle of the electron and the angle $\gamma_{H}$ of a massless object balancing the momentum vector of the electron to satisfy energy and momentum conservation. In the naive quark-parton model $\gamma_{H}$ would be the scattering angle of the struck quark. It is determined from the hadronic energy flow measured in the detector $[20]$,

$\cos \gamma_{H}=\frac{\left(\sum_{i} p_{X}\right)^{2}+\left(\sum_{i} p_{Y}\right)^{2}-\left(\sum_{i}\left(E-p_{Z}\right)\right)^{2}}{\left(\sum_{i} p_{X}\right)^{2}+\left(\sum_{i} p_{Y}\right)^{2}+\left(\sum_{i}\left(E-p_{Z}\right)\right)^{2}}$,

where the sums run over all calorimeter cells $i$, excluding those assigned to the scattered electron, and $\boldsymbol{p}=$ $\left(p_{X}, p_{Y}, p_{Z}\right)$ is the momentum vector assigned to each cell of energy $E$. The cell angles are calculated from the geometric center of the cell and the vertex position of the event. Final state particles produced close to the direction of the proton beam give a negligible contribution, since these particles have $\left(E-p_{Z}\right) \sim 0$.

The invariant mass of the hadronic system detected in the calorimeter, $M_{\mathrm{X}}$, can be determined from the 
cell information. We chose to apply an approach $\# 3$ similar to the double angle method to determine $M_{\mathrm{X}}$.

For one of the experimental cuts to be discussed later, we chose the Jacquet-Blondel estimator of the variable $y$ determined using hadronic measurements only,

$y_{\mathrm{JB}}=\sum_{i}\left(E-p_{Z}\right) / 2 E_{e}$,

where the sum runs over all calorimeter cells $i$ excluding those assigned to the electron.

An important variable used to identify the neutral current deep inelastic events is the quantity $\delta$ defined by

$\delta=\sum_{i}\left(E-p_{Z}\right)$,

where the sum runs over all calorimeter cells $i$. For fully contained neutral current DIS events, and neglecting detector smearing effects, $\delta=2 E_{e}$, where $E_{e}$ is the energy of the incoming electron.

Our choice of technique to determine kinematic variables based on angular quantities reduces systematic effects due to the presence of inactive material between the interaction point and the calorimeter. It does not affect any conclusion presented in this paper.

\section{Triggering and data selection}

To trigger on neutral current DIS events we required signals above predefined thresholds in the EMC cells of the RCAL and BCAL [20]. The triggers were rejected if they were in coincidence with a signal from the beam background counter, located upstream of the detector, in time with the proton beam. Other backgrounds were rejected by cuts on the FCAL and

\#3 If we denote by $E_{H}, p_{H}$ and $\theta_{H}$, the energy, the momentum and the polar angle of the final hadronic system observed in the detector, respectively, then the following formulae determine $M_{\mathrm{X}}: \cos \theta_{H}=\sum_{i} p_{Z} /\left|\sum_{i} p\right|$, where the sum runs over all calorimeter cells $i$, excluding those assigned to the electron, $E_{H}-p_{H} \cos \theta_{H}=$ $2 E_{e} y_{\mathrm{DA}}, p_{H}^{2} \sin ^{2} \theta_{H}=Q_{\mathrm{DA}}^{2}\left(1-y_{\mathrm{DA}}\right), M_{\mathrm{X}}=\left(E_{H}^{2}-\right.$ $\left.p_{H}^{2}\right)^{1 / 2}$. These formulae hold because the undetected particles in the FCAL beam hole have a negligible effect on the determination of the DA variables.
RCAL times. The trigger acceptance for neutral current events, studied both with data and by Monte Carlo techniques, increases with $Q^{2}$ and exceeds $98 \%$ for $Q^{2}>10 \mathrm{GeV}^{2}$.

Further selection criteria were applied in the off-line analysis. The key signature of a neutral current event is the presence of the scattered electron in the final state. The electron identification algorithm is based on the lateral and longitudinal profiles of energy depositions in the calorimeter characteristic of electromagnetic showers. The efficiency was $97 \%$ for identifying electrons with energy greater than $5 \mathrm{GeV}$, as determined from a Monte Carlo simulation of DIS interactions. Events for which no electron with energy above $5 \mathrm{GeV}$ was identified were rejected from the sample.

As stated previously, the variable $\delta$ is expected to be close to $2 E_{e}=53 \mathrm{GeV}$ for neutral current DIS events. Undetected particles emitted in the backward direction and escaping through the rear beam hole, including photons from initial state radiation off the electron, decrease $\delta$. Beam associated backgrounds remaining after timing selections tend to have small values of $\delta$. Photoproduction events, in which the final state electron goes into the RCAL beam hole, also give a small $\delta$. Events for which $\delta$ was less than $35 \mathrm{GeV}$ were rejected. This cut removes remaining beam induced background as well as most of the background from photoproduction.

The electron polar angle was measured with a typical resolution of $5 \mathrm{mrad}$ using the calorimeter information and the event vertex. To ensure full energy containment of the electron shower, events were rejected if the scattered electron position in the RCAL was reconstructed within a square of $32 \mathrm{~cm} \times 32 \mathrm{~cm}$ centered on the beam axis.

The resolution in $\gamma_{H}$ depends on the value of $y_{\mathrm{JB}}$. At small values of $y_{\mathrm{JB}}$, when the current jet is emitted in the direction of the incoming proton, the resolution in $\gamma_{H}$ becomes poor. We thus require $y_{\mathrm{JB}}$ to be greater than 0.04 . In addition, in order to reduce acceptance corrections and background, we select only those events for which $Q_{\mathrm{DA}}^{2}$ is greater than $10 \mathrm{GeV}^{2}$.

Cuts were applied to remove elastic QED Compton events and cosmic muon events based on the pattern of energy deposits in the calorimeter cells, calorimeter timing, and tracking information. After all selections, 1441 events remained. 


\section{Monte Carlo simulation}

The detector acceptance and performance were studied extensively with a Monte Carlo simulation of the experiment. Neutral current DIS events with $Q^{2}>4 \mathrm{GeV}^{2}$ were generated with HERACLES [21], which includes electroweak radiative corrections to first order. The hadronic final state was simulated using the color dipole model [22] as implemented in ARIADNE [23] for the QCD cascade and the Lund string model using JETSET [24] for the soft hadronization process. The results of the simulation are representative of other models based on the Lund string approach and describe well the general trends observed in the final hadronic state of neutral current DIS events [2] ${ }^{\# 4}$. For the parametrization of parton densities in the proton we have used the MRSD-set [25]. The detector simulation is based on the general purpose program GEANT3.13 [26] and incorporates our best knowledge of the experimental environment and trigger. We have generated a sample of events which, after applying the same selection criteria as for the data, yield seven times the number of events in the data sample.

\section{Observation of events with a large rapidity gap}

Most of the characteristics of the 1441 events are similar to those of the Monte Carlo DIS events. In one important aspect, however, the data and Monte Carlo events are different. In the data we observe a substantial number of events with low energy deposited in FCAL $\left(E_{\mathrm{FCAL}}<1 \mathrm{GeV}\right)$ in contrast to the Monte Carlo expectations. In the Monte Carlo sample, events with low $E_{\mathrm{FCAL}}$ occur predominantly at low $y$ and do not pass the cut $y_{\mathrm{JB}}>0.04$. In the data we find events with low $E_{\mathrm{FCAL}}$ that have relatively high $y_{\mathrm{JB}}$, with values extending up to $y_{\mathrm{JB}} \sim 1$. Large values of $y$ at low $x$ correspond to interactions in which the current jet is emitted in the direction of the exchanged virtual photon; that is, at a large angle with respect to the original proton direction. The color transfer between the struck quark and the proton remnant is responsible for

\#4 Note that, in the study reported in ref. [2], events for which the energy deposited in the forward part of the calorimeter was less than $1 \mathrm{GeV}$ were removed.
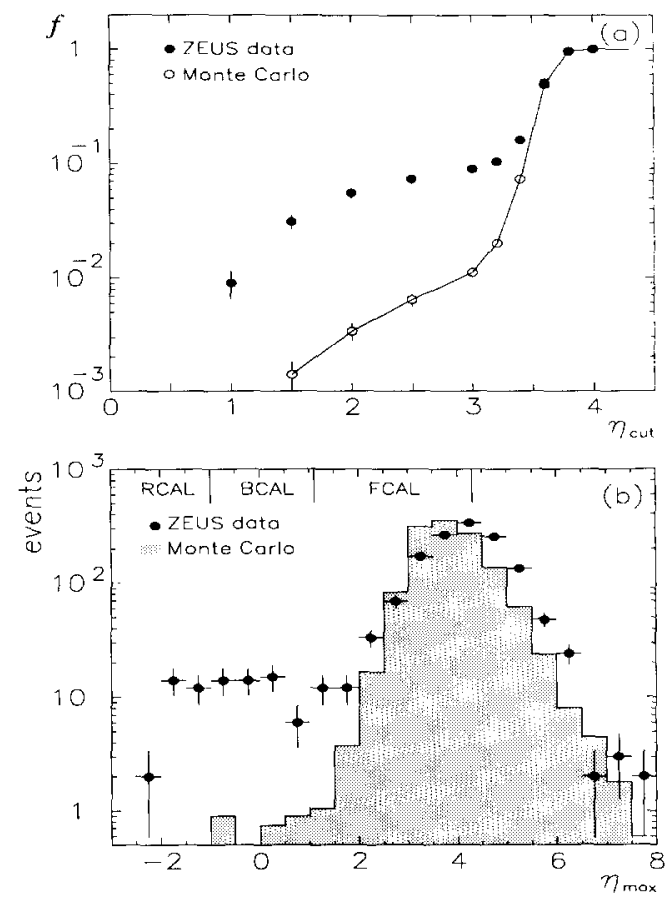

Fig. 2. (a) The fraction $f$ of events for which $99 \%$ of the hadronic energy is contained within the pseudorapidity interval $-3.8<\eta<\eta_{\text {cut }}$ as a function of $\eta_{\text {cut }}$, for data and for Monte Carlo events. (b) Distribution of $\eta_{\max }$, the maximum rapidity of a calorimeter cluster in an event, where a cluster is defined as an isolated set of adjacent cells with summed energy higher than $400 \mathrm{MeV}$, for data and for Monte Carlo events. The boundaries of the calorimeter are indicated. Values of $\eta_{\max }>4.3$ may occur when particles are distributed in many contiguous cells around the beam hole of the FCAL. The number of Monte Carlo events with $\eta_{\max }>1.5$ is normalized to the number of data events in the same $\eta_{\max }$ range.

populating the rapidity interval between them with final state hadrons [3]. Monte Carlo events, therefore, have a substantial energy deposit in FCAL.

Fig. 2a shows for both data and Monte Carlo the fraction of events, $f$, for which $99 \%$ of the hadronic energy measured in the calorimeter is contained within $-3.8<\eta<\eta_{\text {cut }}$, as a function of $\eta_{\text {cut. }}$ The lower limit of $\eta=-3.8$ corresponds to the largest measurable polar angle in the detector, defined by the inner edge of RCAL. Note that the $\eta_{\text {cut }}$ range covers the angular acceptance of the FCAL. In almost all Monte Carlo events, the full calorimeter acceptance is required to measure $99 \%$ of the deposited energy. However, in the data we observe a clear excess of 
events (over Monte Carlo) for which $99 \%$ of the deposited energy is contained within a smaller angular range, corresponding to $\eta_{\mathrm{cut}}<3$.

To study these events further, we define $\eta_{\max }$ as the maximum pseudorapidity of all calorimeter clusters in an event, where a cluster is defined as an isolated set of adjacent cells with summed energy higher than $400 \mathrm{MeV}$. The distribution of $\eta_{\max }$ is presented in fig. $2 b$ and is compared to the distribution obtained from the Monte Carlo simulation. Values of $\eta_{\max }>4.3$ are an artifact of the clustering algorithm and may occur when particles are distributed in many contiguous cells around the beam hole. Note that in addition this region of large $\eta_{\max }$ is particularly sensitive to the details of the proton remnant fragmentation. A clear excess of events is observed for $\eta_{\max }<1.5$. In the data, 89 of the 1441 events have $\eta_{\max }<1.5$, while only $4 \pm 1$ are expected from the simulation of DIS events $\# 5$. We denote events with $\eta_{\max }<1.5$ as events with a large rapidity gap. This corresponds to requiring a rapidity gap of at least 2.8 units.

We have estimated the number of background events in this sample of events with a large rapidity gap. From a Monte Carlo simulation of electrongas interactions we calculate a background of $7 \pm 3$ events. This is consistent with the observation that one event from the unpaired electron bunch survives all the selection cuts. We have scanned all events in the sample and found no event due to cosmic muons or QED Compton interactions. Thus an excess of $78 \pm 10$ events remains, corresponding to $5.4 \%$ of the DIS sample.

\section{Properties of events with a large rapidity gap}

Deep inelastic interactions due to diffractive virtual photon-proton scattering $\left(\gamma^{*} p\right)$ are expected to produce events with a large rapidity gap in the final hadronic state. In the triple pomeron description [5] of diffractive scattering one expects a flat rapidity distribution of the recoiling diffractive state. For fully

\#5 The probability of producing an event with a rapidity gap of greater than 2 units by effects in perturbative QCD has been estimated for quark antiquark pair production at LEP energies to be below $10^{-3}[27]$, consistent with measurements at lower energies [28].
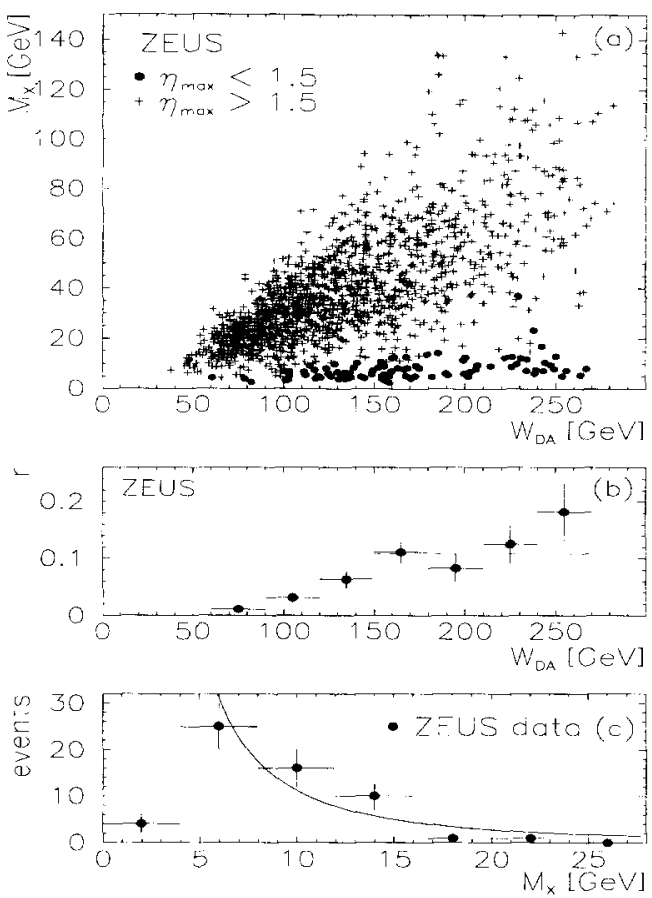

Fig. 3. (a) Correlation between the invariant mass $M_{\mathrm{X}}$ of the hadrons observed in the calorimeter and the invariant mass, $W_{\mathrm{DA}}$, of the $\gamma^{*} p$ system. Events with a large rapidity gap, $\eta_{\max }<1.5$, are shown as solid dots, and events with $\eta_{\max }>1.5$ are shown as crosses. (b) Fraction of events $r$ with a large rapidity gap in the DIS sample as a function of $W_{\mathrm{DA}}$. The dashed line indicates the region in which acceptance corrections are independent of $\boldsymbol{W}$. (c) Distribution of $M_{\mathrm{X}}$ for events with a large rapidity gap, $\eta_{\max }<1.5$, and $W_{\mathrm{DA}}>150 \mathrm{GeV}$. The solid line shows a $1 / M_{\mathrm{X}}^{2}$ dependence. The lowest $M_{\mathrm{X}}$ point is strongly affected by acceptance and detector smearing.

contained diffractive states the $\eta_{\max }$ variable is related to the rapidity distribution of the hadronic final state, neglecting effects due to fragmentation and selection cuts. In the region of $\eta_{\max }<1.5$ in fig. $2 b$, the measured distribution is indeed flat. Motivated by the flatness of the $\eta_{\max }$ distribution we have looked into the inclusive properties of the large rapidity gap events.

We divide the DIS events into two samples, one with $\eta_{\max }<1.5$ and the other with $\eta_{\max }>1.5$. The following conclusions are not sensitive to the exact value chosen. In fig. 3 a we present for both samples the correlation between the invariant mass $M_{\mathrm{X}}$ of the observed hadronic system in the detector and the total available energy $W_{\mathrm{DA}}$ in the $\gamma^{*} p$ system. A feature of the events with a large rapidity gap is that $M_{\mathrm{X}}$ is 


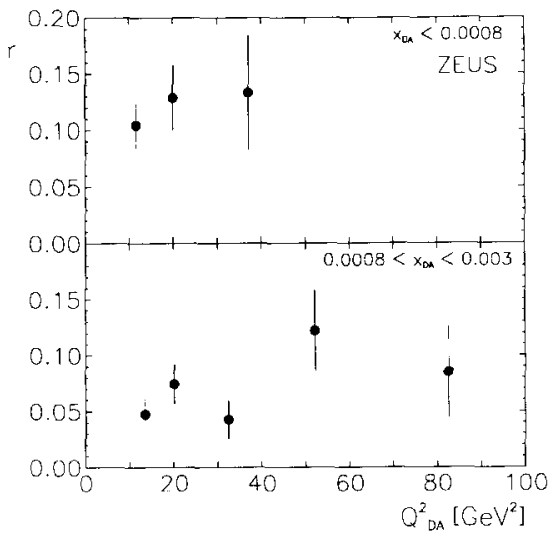

Fig. 4. Fraction $r$ of events with a large rapidity gap, $\eta_{\max }<1.5$, as a function of $Q_{\mathrm{DA}}^{2}$ for two ranges of $x_{\mathrm{DA}}$. No acceptance corrections have been applied.

small compared to $W_{\mathrm{DA}}$ and is typically smaller than $10 \mathrm{GeV}$. The events span the range of $W_{\mathrm{DA}}$ from 60 to $270 \mathrm{GeV}$. For $W_{\mathrm{DA}}>150 \mathrm{GeV}$ these events are well separated from the rest of the sample. In this region, acceptance corrections have little dependence on $W$ and the contribution of these events to the deep inelastic cross section is, within errors, constant with $W_{\mathrm{DA}}$, as expected for a diffractive type of interaction (see fig. 3b). At smaller values of $W_{\mathrm{DA}}$, the acceptance for these events decreases since the final state hadronic system is boosted in the forward direction.

In fig. $3 c$ we present the distribution of $M_{\mathrm{X}}$ for events with $\eta_{\max }<1.5$ and $W_{\mathrm{DA}}>150 \mathrm{GeV}$. The distribution is not corrected for detector or acceptance effects. Although this acceptance could be model dependent, the three models we have checked $[13,14,16]$ predict a flat acceptance with $M_{\mathrm{X}}$ for $M_{\mathrm{X}}>4 \mathrm{GeV}$. We observe a spectrum which, given our resolution, the uncertainty about the acceptance and the large statistical errors, is compatible with a $1 / M_{\mathrm{X}}^{2}$ dependence, shown as the solid curve.

The fraction of events with a large rapidity gap, presented as a function of $Q_{\mathrm{DA}}^{2}$ in fig. 4 for two selected bins of $x_{\mathrm{DA}}$, is, within errors, independent of $Q^{2}$. The $Q^{2}$ dependence is little affected by acceptance corrections. In QCD terminology, leading twist contributions to structure functions show little (at most logarithmic) dependence on $Q^{2}$ at fixed $x$, whereas higher twist terms fall as a power of $Q^{2}$. Since the proton structure function determined for our DIS data sample shows a leading twist behavior [29], the produc- tion mechanism responsible for the large rapidity gap events is also likely to be a leading twist effect. The decrease with $x$ is partly due to acceptance, since for larger values of $x$ the final hadronic state is boosted in the direction of the proton so that such events will not be identified as having a large rapidity gap in our detector.

\section{Discussion and conclusions}

In a sample of deep inelastic neutral current scattering events, we have observed a class of events with a large rapidity gap in the final hadronic state. The flat rapidity distribution, the lack of $W$ dependence and the shape of the $M_{\mathrm{X}}$ distribution are suggestive of a diffractive interaction between a highly virtual photon and the proton, mediated by the exchange of the pomeron [5]. The fact that the percentage of events with a large rapidity gap shows only a weak dependence on $Q^{2}$ points to a leading twist contribution to the proton structure function.

For the hypothesis that events with a large rapidity gap are produced by a diffractive mechanism, one expects such events to be accompanied by a quasielastically scattered proton. For this type of process the gap between the maximum rapidity of the calorimeter and the rapidity of the scattered proton is about three units. The selection criteria, in particular the requirement of a rapidity gap in the detector of at least 2.8 units, limit the acceptance for diffractivelike events. Since we have made no corrections for acceptance, the $5.4 \%$ for DIS events with a large rapidity gap should be considered a lower limit for diffractively produced events.

\section{Acknowledgement}

We wish to acknowledge the contribution of the HERA machine staff during the first year of operating this new colliding beam facility. The support of the DESY directorate is greatly appreciated. We also acknowledge most informative discussions with J. Bartels, J. Ellis, G. Ingelman, E. Levin, and B. Webber. 


\section{References}

[1] H1 Collab., T. Ahmed et al., Phys. Lett. B 298 (1993) 469.

[2] ZEUS Collab., M. Derrick et al., Z. Phys. C 59 (1993) 231 , and references therein.

[3] B. Andersson, G. Gustafson and T. Sjöstrand, Phys. Lett. B 94 (1980) 211.

B. Andersson, G. Gustafson, G. Ingelman and T. Sjöstrand, Phys. Rep. 97 (1983) 31;

B.R. Webber, Nucl. Phys. B 238 (1984) 492;

Ya.I. Azimov, Yu.L. Dokshitzer, V.A. Khoze and S.I. Troyan, Phys. Lett. B 165 (1985) 147.

[4] E.L. Berger, J.C. Collins, D.E. Soper and G. Sterman, Nucl. Phys. B 286 (1987) 704.

[5] R.D. Field and G.C. Fox, Nucl. Phys. B 80 (1974) 367 ;

A.B. Kaidalov and K.A. Ter-Martirosyan, Nucl. Phys. B 75 (1974) 471;

A. Donnachie and P.V. Landshoff, Nucl. Phys. B 231 (1984) 189; B 267 (1986) 690.

[6] K. Goulianos, Phys. Rep. 101 (1983) 169; Nucl. Phys. B, Proc. Suppl. 12 (1990) 110.

[7] G. Ingelman and P.E. Schlein, Phys. Lett. B 152 (1985) 256.

[8] UA8 Collab., R. Bonino et al., Phys. Lett. B 211 (1988) 239 ;

A. Brandt et al., Phys. Lett. B 297 (1992) 417.

[9] L.V. Gribov, E.M. Levin and M.G. Ryskin, Phys. Rep. 100 (1983) 1.

[10] J. Bartels and G. Ingelman, Phys. Lett. B 235 (1990) 175.

[11] E.M. Levin and M. Wüsthoff, DESY 92-166, FERMILAB-Pub-92/334.

[12] M.G. Ryskin, Sov. J. Nucl. Phys. 53 (1991) 668.
[13] G. Ingelman and K. Prytz, Z. Phys. C 58 (1993) 285.

[14] N.N. Nikolaev and B.G. Zakharov, Z. Phys. C 53 (1992) 331.

[15] J.C. Collins, L. Frankfurt and M. Strikman, Phys. Lett. B 307 (1993) 161.

[16] A. Donnachie and P.V. Landshoff, Phys. Lett. B 191 (1987) 309.

[17] K.H. Streng, Proc. HERA Workshop, Vol. 1, ed. R.D. Peccei (1987) p. 365; CERN-TH.4949 (1988).

[18] ZEUS Collab., M. Derrick et al., Phys. Lett. B 293 (1992) 465 .

[19] S. Bentvelsen, J. Engelen and P. Kooijman, Proc. Workshop on Physics at HERA, Vol. 1, eds. W. Buchmüller and G. Ingelman (1991) p. 23.

[20] ZEUS Collab., M. Derrick et al., Phys. Lett. B 303 (1993) 183.

[21] A. Kwiatkowski, H. Spiesberger and H.-J. Möring, Proc. Workshop on Physics at HERA, Vol. 3, eds. W. Buchmüller and G. Ingelman (1991) p. 1294.

[22] B. Andersson, G. Gustafson, L. Lönnblad and U. Pettersson, Z. Phys. C 43 (1989) 625.

[23] L. Lönnblad, LU TP-89-10 (1989).

[24] T. Sjöstrand, Comput. Phys. Commun. 39 (1986) 347; T. Sjöstrand and M. Bengtsson, Comput. Phys. Commun. 43 (1987) 367.

[25] A.D. Martin, W.J. Stirling and R.G. Roberts, Phys. Rev. D 47 (1993) 867.

[26] R. Brun et al., CERN DD/EE/84-1 (1984).

[27] J.D. Bjorken, S.J. Brodsky and H.J. Lu, Phys. Lett. B 286 (1992) 153;

H.J. Lu, S.J. Brodsky and V.A. Khoze, SLAC-PUB6113 (1993).

[28] M. Derrick et al., Z. Phys. C 35 (1987) 323.

[29] ZEUS Collab., M. Derrick et al., Measurement of the proton structure function $F_{2}$ in $e p$ scattering at HERA, to be published. 